\title{
Elektronische Abrechnung unter TARMED
}

\author{
Dr. med. Jacques de Haller ${ }^{\text {, Dr. med. A. Haefeli }}{ }^{b}$, Dr. med. F. Muggli ${ }^{c}$
}

Nur 15 Monate nach Inkraftsetzung des TARMED rechnen bereits $80 \%$ der freipraktizierenden Ärztinnen und Ärzte elektronisch ab. Sie erfüllen damit die vertraglichen Bestimmungen zum elektronischen Abrechnen bereits weit vor dem 1. Januar 2006. Dennoch bestehen rund um das Thema der elektronischen Abrechnung viele Missverständnisse, offene Fragen und leider auch Unsicherheiten. Nachstehend wird die Position der kantonalen Ärztegesellschaften und der FMH in Sachen elektronische Abrechnung erklärt.

\section{Rahmenbedingungen KVG und TARMED}

Mit dem einheitlichen Arzttarif TARMED hat auch der Begriff der elektronischen Abrechnung Einzug gehalten. Dieser wird sowohl im KVG wie auch im Tarif selber verwendet. Leider oft aber auch missverstanden.

Art 42 Abs. 3 KVG

hält fest, dass der Leistungserbringer dem Schuldner eine detaillierte und verständliche Rechnung zustellen muss. Es besteht daher eine Bringschuld für die Zustellung der Rechnung an den Schuldner.

\section{TARMED}

Der TARMED definiert in den generellen Interpretationen (GI-50) die elektronische Abrechnung wie folgt: «Das Kriterium der «lektronischen Abrechnung> ist dann erfüllt, wenn die Rechnung dem Versicherer nach dem von den jeweiligen Vertragspartnern vereinbarten Standard elektronisch übermittelt wird.»

Der TARMED enthält in der technischen Leistung eine Abgeltung für die Personal-, Sachund Infrastrukturkosten. Eine spezifische Position für die elektronische Rechnungsstellung gibt es nicht.

\section{Feststellung der FMH und der Kantonalen} Ärztegesellschaften (G7)

a) Die generelle Interpretation zur elektronischen Abrechnung ist auf das Tierspayant-System spezifiziert. Im «Tiers garant» stellt der Leistungserbringer seine Rechnung immer direkt den Patienten zu. Die GI-50 kann in den Kantonen mit einer Rechnungsstellung an den Patienten (Tiers garant) nicht einen Zwang zur Umstellung der Fakturierung in das System des «Tiers payant» (Rechnung an Versicherer) herbeiführen. Eine b Präsident G7

c Leiter Taskforce TARMED FMH

\section{Zusammenfassung}

Dank eines speziell für das System des «Tiers garant» entwickelten Verfahrens können die Versicherer nach Einreichung der Rechnung durch die Versicherten vollautomatisch eine elektronische Kopie der Rechnung bei einem der dazu aufgebauten TrustCenter abrufen. Sie sparen damit Zeit und Kosten für die Erfassung und die Kontrolle der Rechnungen. Da im Transport aus den Praxen bis zu den Krankenversicherern keine Medienbrüche mehr bestehen, ist die Qualität der elektronischen Daten zudem fehlerfrei. Diese Dienstleistungen stellen für die Versicherer echte Mehrwerte dar. Und sie ermöglichen ihnen namhafte Einsparungen, da damit personalintensive Verarbeitungsprozesse in der Rechnungsverarbeitung und -kontrolle optimiert werden können.

solche Umstellung bedingt nach KVG eine bilaterale Vereinbarung der Parteien.

b) Die Zuständigkeit zur Bestimmung des Abrechnungsverfahrens liegt bei den Tarifpartnern. Können sich diese nicht einigen, bestimmt das KVG den «Tiers garant» als Standard. Die geltenden, von den kantonalen Behörden genehmigten TARMED-Anschlussverträge $(\mathrm{AV})$ sind daher zu respektieren.

c) Aufgrund der kantonalen Anschlussverträge ist ein Wechsel vom «Tiers garant» zum «Tiers payant» für die einzelne Ärztin / den einzelnen Arzt nur nach einer Kündigung oder Änderung der Anschlussverträge möglich. Anderslautende Aussagen sind sowohl für die Leistungserbringer wie für die Patientinnen und Patienten irreführend.

\section{Pflichten der Ärztin / des Arztes im System «Tiers garant»}

Gemäss dem Rahmenvertrag TARMED (RV) und den kantonalen Anschlussverträgen (AV) ist die Ärztin / der Arzt, welche/welcher direkt mit seinen Patientinnen und Patienten abrechnet, verpflichtet, eine elektronische Kopie der Rechnung für den Versicherer «bereitzustellen». 
Art. 11 RV:

\section{Rechnungsstellung und Vergütung}

Anhang 3 RV Ziff. 2 Rechnungsstellung enthält folgenden Absatz:

«Die Vertragsparteien verpflichten sich, die Vorgaben betreffend einheitlichem Rechnungsformular elektronischer Rechnungsstellung und Weitergabe medizinischer Daten gemäss Art. 11 Abs. 4, 5 und 6 sowie Art. 14 Abs. 2 des TARMED-Rahmenvertrages umzusetzen.

Die inhaltliche und technische Umsetzung und die Regelung über die Kostentragung erfolgt auf Basis der gemeinsam erarbeiteten Standards und Richtlinien im Rahmen des Forums für den elektronischen Informationsund Datenaustausch im Gesundheitswesen $[\ldots]$.»

Wie die Bereitstellung der Daten und diese Umsetzung gemäss Anhang 3 vor sich gehen sollen, ist bis heute nicht geregelt.

\section{Feststellung der FMH und der kantonalen Ärztegesellschaften (G7)}

a) Rund $80 \%$ der freipraktizierenden Ärztinnen und Ärzte erstellen ihre Rechnungen heute elektronisch. Diese stehen daher in den Praxen bereit.

b) Über $60 \%$ der freipraktizierenden Ärztinnen und Ärzte haben sich zudem freiwillig einem Intermediär angeschlossen (MediData, TrustCenter usw.), welche die elektronischen Rechnungskopien aus den Praxen sammeln, sicher aufbewahren und für die internetbasierte, datenschutzkonforme zentrale Bereitstellung für die Versicherer sorgen.

c) Die gemeinsame Verhandlungsdelegation der FMH/G7 und der santésuisse hat im Herbst 2003 eine Vereinbarung zur Regelung der Modalitäten im elektronischen Austausch der Rechnungsdaten erarbeitet. Diese wurde seitens der kantonalen Ärztegesellschaften genehmigt. Santésuisse hat sich gegen eine Verbandslösung ausgesprochen und den Vertrag nicht genehmigt.

d) Die Verweigerung einer Verbandslösung ohne einen konkreten Gegenvorschlag hat einzelne Krankenversicherer und Intermediäre gezwungen, selber Wege zur Lösung des eDA zu suchen. Diese wurden inzwischen erfolgreich getestet und realisiert. Der elektronische Austausch von Rechnungsdaten wird daher heute produktiv genutzt und hat sich in der Praxis bewährt.
Die freipraktizierenden Ärztinnen und Ärzte haben damit im Bereich der elektronischen Abrechnung ihre Hausaufgaben gemacht. Sie rechnen zu 80\% elektronisch ab und erfüllen damit die Prämissen der elektronischen Abrechnung und zur Aufhebung der Limitationen. Darüber hinaus haben sie den Krankenversicherern Wege zur Abholung der bereitgestellten Rechnungsdaten durch die Schaffung der TrustCenter vorbereitet. Dies freiwillig und auf eigene Kosten.

FMH und G7 sind der Auffassung, dass alle Ärztinnen und Ärzte, welche ihre Daten in einem TrustCenter oder bei einem anderen Intermediär «bereitstellen», die Bedingungen für die elektronische Abrechnung über die minimale Bereitstellung hinaus erfüllen. Damit sind diese Ärztinnen und Ärzte von den Limitationen zu befreien.

\section{Abgeltung von Dienstleistungen der Intermediäre}

Mit Schreiben vom 8. Februar 2005 hält Dr. med. H. H. Brunner, Vizedirektor BAG, folgende Punkte zu diesem Thema fest.

- für die Rechnungsstellung darf keine Gebühr erhoben werden. Der Leistungserbringer muss dem Schuldner die Rechnung ohne zusätzliche Gebühren zustellen.

- den Krankenversicherern wird freigestellt, Verträge für echte zusätzliche Dienstleistungen (z.B. von Intermediären) zur Abholung der «bereitgestellten» TARMED-Rechnungen einzugehen, um Verwaltungskosten zu sparen oder die Kontrolle der Rechnungen zu optimieren.

\section{Feststellung der FMH und der kantonalen Ärztegesellschaften (G7)}

a) Die Ärzteschaft hat unter der Federführung der Firma NewIndex über die gesamte Schweiz elf TrustCenter aufgebaut. Diese sammeln bereits heute «bereitgestellte» TARMED-Rechnungen aus über 7000 Praxen ein und halten sie zentral zur Nutzung durch die Krankenversicherer bereit. Die Versicherer können sich damit den Aufwand für die Sammlung und Zusammenführung der Daten aus den vielen Praxen ersparen. Es ist davon auszugehen, dass die Krankenversicherer jährlich über 20 Mio. Franken einsparen könnten, wenn sie die Dienste der Intermediäre nutzen würden. 
b) Beim Aufbau der TrustCenter wurde speziell auf die gesetzlichen Auflagen der ärztlichen Schweigepflicht und die Bestimmungen des Datenschutzgesetzes geachtet. Die Rechnungsdaten werden daher bereits in den Arztpraxen pseudonomysiert und für den Transport über das Internet verschlüsselt. Die Versicherer profitieren dabei vom Know-how und der Sorgfalt in der Umsetzung der technischen Lösung durch die TrustCenter. Sie können die elektronischen Rechnungsdaten datenschutzkonform und ohne eigene Investitionen von den TrustCentern abholen.

c) Leider werden die TARMED-Rechnungen von den Versicherern nur zu einem geringen Anteil «abgeholt» und genutzt. Das Einsparungspotential dieser vollelektronisch vorliegenden Daten liegt damit ungenutzt brach.

- Die praktizierenden Ärztinnen und Ärzte sind in der Lage, dem Honorarschuldner (meistens dem Patienten) detaillierte Rechnungen zuzustellen.
- Die meisten Ärztinnen und Ärzte sind in der Lage, mit der eingesetzten Praxissoftware elektronische Rechnungskopien im festgelegten Standard $\mathrm{zu}$ erzeugen und für die Versicherer in den Trustcentern zum Abholen bereitzustellen. Mit dieser datenschutzkonformen, korrekten Bereitstellung und Aufbewahrung, bereits in Pilotprojekten erprobten, einwandfrei funktionierenden Übermittlung der vollständigen Datensätze an die Versicherer schaffen wir ein hohes Nutzenpotential. Die Versicherer können dieses umsetzen. Die Erfassung, administrative Bearbeitung, Plausibilisierung der Rechnungsinhalte, wird vereinfacht.

Wir fordern die Versicherer auf, diesen Nutzen anzuerkennen und sich das damit verbundene Einsparpotential zu erschliessen. 